\title{
Innovative and Efficient Strategy of Calibrating Sentinel-1
}

\author{
Marco Schwerdt, Benjamin Bräutigam, Björn Döring, Manfred Zink \\ German Aerospace Center (DLR), Microwaves and Radar Institute \\ Oberpfaffenhofen, D-82234 Wessling, Germany \\ Tel.: +49 8153-283112, Fax: +49 8153-281449 \\ marco.schwerdt@dlr.de
}

\section{Introduction}

As part of the GMES system, Sentinel-1 is designed to provide an independent and operational information capacity to the European Union to warrant environment and security policies and to support sustainable economic growth. Sentinel- 1 is a satellite system designed to operate a ground segment for 20 years supporting a system of up to three satellites. One satellite is built to perform at least seven years in the reference orbit and to operate a SAR instrument in Cband. Product quality is of paramount importance. Hence, the success or failure of the mission is essentially dependent on the calibration of the Sentinel- 1 system ensuring the product quality and the correct in-orbit operation of the entire SAR system.

The essential task of calibrating Sentinel-1 is to estimate and correct systematic error contributions throughout the complete SAR system and to tie-down image information (magnitude and phase) to reference units in geophysical terms. The quality of this calibration process is dependent on the inherent stability of the radar system and the capability to determine and monitor the radiometric and geometric characteristics.

\section{Calibration Strategy}

The time frame for performing all calibration activities is defined by the tight duration of the commissioning phase of Sentinel-1. Considering a repeat cycle of 12 days, 3 months commissioning phase results in 7.5 repeat cycles. Assuming half a cycle for the check out of the complete Sentinel-1 System including both space and ground segment as well as one cycle for product release at the end of commissioning Sentinel-1, 6 repeat cycles remain for performing all calibration activities. The minimum number of measurements being performed and consequently of passes being required is driven by the radiometric accuracy budget and the strategy to execute measurements on selected beams.

This strategy is based on two key elements, an accurate internal calibration facility for monitoring and characterizing the whole instrument down to individual transmit/receiver modules and a precise antenna model shifting most of the antenna characterization from the commissioning phase to pre-launch activities. Thus, after launch only a set of suitable beams has to be really measured in-flight, ensuring the tight schedule of commissioning Sentinel- 1 .

\section{In-Orbit Calibration Plan}

Nevertheless, due to the high demand on the radiometric accuracy of $1 \mathrm{~dB}(3 \sigma)$ in all 4 operation modes, it is recommended to measure at least:

- one beam of each mode,

- each beam being selected measuring twice (ascending/descending orbit),

- against three reference targets deployed within the swath,

- one beam with low, one with mid and one with high incidence angle on order to cover the wide range of swath positions,

- one beam in both transmit polarizations 
Considering these aspects, one test site with 3 transponders and 3 corner reflectors deployed at mid latitude and within an cross over area of ascending and descending swaths is sufficiently to cope with the tight requirements of commissioning Sentinel-1.

The paper will described the strategy and the in-orbit calibration plan for performing all calibration procedures required in an efficient way. This external calibration scenario demonstrates the capability to perform the different calibration procedures within the tight schedule of 3 months commissioning phase of Sentinel-1. 